1997-08-22

\title{
A fluid in contact with a semipermeable surface: Second-order integral equation approach
}

Douglas Henderson

Pawel Bryk

Stefan Sokolowski

Follow this and additional works at: https://scholarsarchive.byu.edu/facpub

Part of the Biochemistry Commons, and the Chemistry Commons

\section{Original Publication Citation}

Bryk, P., D. Henderson, and S. Sokolowski. "A fluid in contact with a semipermeable surface: Second-order integral equation approach." The Journal of Chemical Physics 17 (1997): 3333-3336.

\section{BYU ScholarsArchive Citation}

Henderson, Douglas; Bryk, Pawel; and Sokolowski, Stefan, "A fluid in contact with a semipermeable surface: Second-order integral equation approach" (1997). Faculty Publications. 663.

https://scholarsarchive.byu.edu/facpub/663 


\title{
A fluid in contact with a semipermeable surface: Second-order integral equation approach
}

\author{
P. Bryk \\ Department for the Modelling of Physico-Chemical Processes, Faculty of Chemistry, MCS University, \\ 20031 Lublin, Poland \\ D. Henderson \\ Department of Chemistry and Biochemistry, Brigham Young University, Provo, Utah 84602
}

S. Sokołowski

Department for the Modelling of Physico-Chemical Processes, Faculty of Chemistry, MCS University, 20031 Lublin, Poland

(Received 3 April 1997; accepted 16 June 1997)

An integral equation approach for a binary hard-sphere mixture interacting with a planar semipermeable wall (membrane) is formulated by using the second-order nonuniform or pair Ornstein-Zernike equation as well as the usual singlet Ornstein-Zernike equation. The results of the pair theory are compared with those obtained from the singlet theory and with computer simulation data. The pair approach is more accurate than the singlet theory. (C) 1997 American Institute of Physics. [S0021-9606(97)52332-4]

Using integral equations, the structure of simple nonuniform fluids can be determined either by using a singlet level ${ }^{1}$ or a pair level theory. ${ }^{2-5}$ The limitations of the singlet approach are well known. In particular, the singlet PercusYevick (PY1) and hypernetted chain (HNC1) theories, when applied to a fluid in contact with a hard wall, predict incorrect values of the contact values of the density profiles. ${ }^{1}$ These approximations also fail in the description of surface phase transitions. ${ }^{6}$ Theories at the pair level, although more demanding computationally, are much more accurate in predicting the fluid structure. ${ }^{2-5,7-10}$

Nonuniformity in the fluid can be caused by the presence of impenetrable walls, walls permeable to all fluid molecules, ${ }^{11-14}$ or walls permeable to some species of the fluid mixture. ${ }^{15-17}$ A semipermeable wall, i.e., a semipermeable membrane, that is permeable only to some species in a fluid mixture, are part of many systems of biochemical interest as well as an important construct of osmotic theory. ${ }^{18,19} \mathrm{~A}$ theory of semipermeable membranes and vesicles, based on an integral equation approach, has been proposed by Zhou and Stell. ${ }^{15,16}$ Their theory extends the singlet level theories for the density profiles of a fluid in contact with an inpenetrable wall ${ }^{1}$ to the case when some particles of the fluid can cross the dividing surface. Also, Zhou and Stell ${ }^{15}$ have discussed the possibility of the application of density functional theories ${ }^{6}$ to study such systems. A next step in the development of more accurate techniques for the description of the fluid structure in the vicinity of a membrane is an application of second-order or pair theories; this is the aim of this study. Because of the preliminary character of this note, we limit ourselves here to the case of planar symmetry and to a simple model for the interparticle forces. Specifically, we formulate the theory for a binary mixture of hard spheres in contact with a membrane, which acts like a hard wall to one kind of species but does not interact with the molecules of the second kind. The results of theoretical predictions are compared with Monte Carlo (MC) data.

We consider a fluid mixture of two species $a$ and $b$ of sizes, $\sigma_{a}$ and $\sigma_{b}=1$ in contact with a semipermeable planar membrane (SPM). The membrane is located at $z=0$; the interaction of the membrane with an $a$ particle is given by

$$
v_{a}(z)=\left\{\begin{array}{cc}
0, & z<0 \\
\infty, & z>0
\end{array} .\right.
$$

This means that particles of species $a$ cannot cross the membrane and that they occupy only the left part of the system. The particles $b$ can move freely; they do not interact with the membrane.

The interaction potential $u_{i j}(r), i, j=a, b$ between fluid species is just a hard core potential

$$
u_{i j}(r)=\left\{\begin{array}{cc}
\infty, & r \leqslant \sigma_{i j} \\
0, & r>\sigma_{i j}
\end{array}\right.
$$

where the additivity of the sizes of the species $a$ and $b$ is assumed.

The second-order nonuniform or pair level OrnsteinZernike equation, defining the direct correlation functions (dcf's) $c_{i j}\left(\mathbf{r}_{1}, \mathbf{r}_{2}\right), i, j=a, b$, in terms of the total correlation functions $h_{i j}\left(\mathbf{r}_{1}, \mathbf{r}_{2}\right)$ and the local densities $\rho_{i}(\mathbf{r})$, has the form $^{1}$

$$
\begin{aligned}
h_{i j}\left(\mathbf{r}_{1}, \mathbf{r}_{2}\right)= & c_{i j}\left(\mathbf{r}_{1}, \mathbf{r}_{2}\right) \\
& +\sum_{n=a, b} \int d \mathbf{r}_{3} h_{i n}\left(\mathbf{r}_{1}, \mathbf{r}_{3}\right) c_{n j}\left(\mathbf{r}_{3}, \mathbf{r}_{2}\right) \rho_{n}\left(\mathbf{r}_{3}\right) .
\end{aligned}
$$

The pair theory requires an additional equation relating $\rho_{i}(\mathbf{r})$ to the pair functions. We use the Lovett-Mou-BuffWertheim equation ${ }^{1,20}$ 


$$
\boldsymbol{\nabla} \ln t_{i}\left(\mathbf{r}_{1}\right)=\sum_{n=a, b} \int d \mathbf{r}_{2}\left[\boldsymbol{\nabla} \rho_{n}\left(\mathbf{r}_{2}\right)\right] c_{i n}\left(\mathbf{r}_{1}, \mathbf{r}_{2}\right) .
$$

In the above, $t_{i}(\mathbf{r})$ is the one-particle cavity function, $\rho_{i}(\mathbf{r})$ $=t_{i}(\mathbf{r}) \gamma_{i}(\mathbf{r})$, and $\gamma_{i}(\mathbf{r})=\exp \left[-v_{i}(\mathbf{r}) / k T\right]$ is the Boltzmann factor.

The set of equations (3) and (4) must be supplemented by a closure relation between the nonuniform two-particle functions. We have chosen the Percus-Yevick (PY) approximation, i.e.,

$$
\begin{aligned}
& h_{i j}\left(z_{1}, z_{2}, R_{12}\right)=\left[f_{i j}\left(r_{12}\right)+1\right] y_{i j}\left(z_{1}, z_{2}, R_{12}\right)-1, \\
& c_{i j}\left(z_{1}, z_{2}, R_{12}\right)=f_{i j}\left(r_{12}\right) y_{i j}\left(z_{1}, z_{2}, R_{12}\right),
\end{aligned}
$$

where $y_{i j}\left(z_{1}, z_{2}, R_{12}\right)$ stands for the two-particle nonuniform cavity function, $f_{i j}\left(r_{12}\right)=\exp \left[-\beta u_{i j}(r)\right]-1$ is the Mayer function, and $r_{12}=\sqrt{\left(z_{1}-z_{2}\right)^{2}+R_{12}^{2}}$. Now, Eqs. (3)-(5) called here the PY2 approximation, form a closed set, which can be solved to yield the one- and two-particle correlation functions.

Assuming that the nonuniform direct correlation function in Eq. (4), $c_{i n}\left(\mathbf{r}_{1}, \mathbf{r}_{2}\right)$, can be approximated by its counterpart for an uniform mixture, $c_{i n}^{0}\left(\left|\mathbf{r}_{1}-\mathbf{r}_{2}\right|\right)$, having the same chemical potentials of both species as the investigated mixture, Eq. (4) reduces to the singlet $\mathrm{HNC} 1$ equation for the density profile ${ }^{15,16}$

$$
\ln t_{i}\left(\mathbf{r}_{1}\right) / \rho_{i}^{0}=\sum_{n=A, B} \int d \mathbf{r}_{2} c_{i n}^{0}\left(\left|\mathbf{r}_{1}-\mathbf{r}_{2}\right|\right)\left[\rho_{i}\left(\mathbf{r}_{2}\right)-\rho_{n}^{0}\right],
$$

where $\rho_{n}^{0}$ are the uniform fluid densities. The PY1 equation for the density profile results from the expansion of the logarithm in Eq. (6) yielding

$$
t_{i}\left(\mathbf{r}_{1}\right) / \rho_{i}^{0}=1+\sum_{n=A, B} \int d \mathbf{r}_{2} c_{i n}^{0}\left(\left|\mathbf{r}_{1}-\mathbf{r}_{2}\right|\right)\left[\rho_{i}\left(\mathbf{r}_{2}\right)-\rho_{n}^{0}\right] .
$$

Both of these singlet equations, Eqs. (6) and (7), have been used by Zhou and Stell ${ }^{15}$ to study the behavior of the fluid in contact with a planar semipermeable membrane. The solution of these equations requires the knowledge of the functions $c_{i n}(r)$; they can be easily evaluated using the method described previously. ${ }^{21}$

The numerical algorithm for the solution of the system (4) -(6) consists of the expansion of the two-particle functions into a Fourier-Bessel series. We omit all the details of the numerical method; they can be found in our earlier publications. ${ }^{2-4}$ Computer simulations of the system have been carried out in canonical ensemble, as described earlier. ${ }^{22}$ In order to apply periodic boundary conditions, two semipermeable membranes were set at $z=-Z L$ (together with its periodic replica at $z=Z L)$ and at $z=0$. The membranes divide the system into two subparts. In the subpart $-Z L<z<0$ both components are present; the subpart $0<z$ $<Z L$ contains only permeable component. The elongation parameter, $Z L$, of the system must be large enough to assure the existence of "bulk" parts in both subsystems. Obviously, for any finite $Z L$ we would rather deal with a collec- tion of slits, but our test calculations ${ }^{22}$ have indicated that accepting $Z L \sim 20 \sigma_{a}$, the errors in the "bulk" density evaluation from the average density at the slit centre are small. Ensemble averages were accumulated over at least $10^{8}$ configurations after equilibrating from random distributions for $10^{7}$ configurations.

In Fig. 1, a comparison of the theoretically predicted and the simulated total density profiles, $\rho_{a}(z)$ and $\rho_{b}(z)$, are shown. To make the plots more transparent, only every second local density value, obtained from computer simulations, has been displayed. The calculations have been carried out assuming that both species have the same size [Fig. 1(a)] and for the ratio sizes of nonpermeable and permeable component equal to 1.5 [Fig. 1(b)] and 2.0 [Fig. 1(c)]. The values of total bulk densities, $\rho^{*}=\rho_{a} \sigma_{a a}^{3}+\rho_{b} \sigma_{b b}^{3}$, in the region $z<0$ were $0.7133,0.8190$, and 0.6818 , respectively. Thus the system with the ratio size 1.5 is the most dense.

Let us discuss some general features that are observed in Fig. 1. The agreement between the profiles of permeable and non-permeable components, predicted by the PY2 theory, and the results of the simulations is good. Only in the nearest vicinity of the wall are slight differences observed; in general, the position of the first minimum and the second maximum of the density profiles are very well reproduced by the theory. Also, the bulk density of the permeable component for $z>0$ agrees almost perfectly with the computer simulation result. For $z<0$, the singlet theories are less accurate than the second-order theory, see especially Fig. 1(b), i.e., the most dense system. The bulk densities for $z>0$, obtained from the singlet theories, are considerably less accurate. The PY1 approximation underestimates, these values, compared with the results of computer simulations. On the other hand, the $\mathrm{HNC1}$ approximations overestimates these values. The singlet theory seems to be better when the size of nonpermeable particles is the same as that of the permeable particles.

Figure 2 shows some examples of the pair distribution functions of the nonpermeable component, evaluated for the size ratio 1 [Fig. 2(a)] and 2 [Fig. 2(b)]. The curves illustrate the correlations between a pair of particles located in the bulk part of the system, located in the plane of the membrane and for a perpendicular configuration of both particles, with one particle lying on the membrane. It can be seen that the location of the subsequent maxima and minima of the pair correlation functions is almost the same. The lateral correlations in the surface plane are slightly weaker, whereas the correlations in the direction perpendicular to the membrane are slightly stronger compared to the correlations in the bulk. For larger interparticle separations, the shape of all three functions is almost the same. Despite the lower bulk density, the differences between the above mentioned three functions are greater when $\sigma_{a a} / \sigma_{b b}=2$ than when $\sigma_{a a} / \sigma_{b b}=1$.

Our calculations indicate that the second-order theory is more accurate in predicting the density distribution of hardsphere particles near a semipermeable membrane than are the singlet theories. Also, the second-order theory gives insight into the nature of the pair correlation functions. An extension of the theory to the case of semipermeable vesicles can be formulated by using the technique developed by Attard. ${ }^{5}$ 

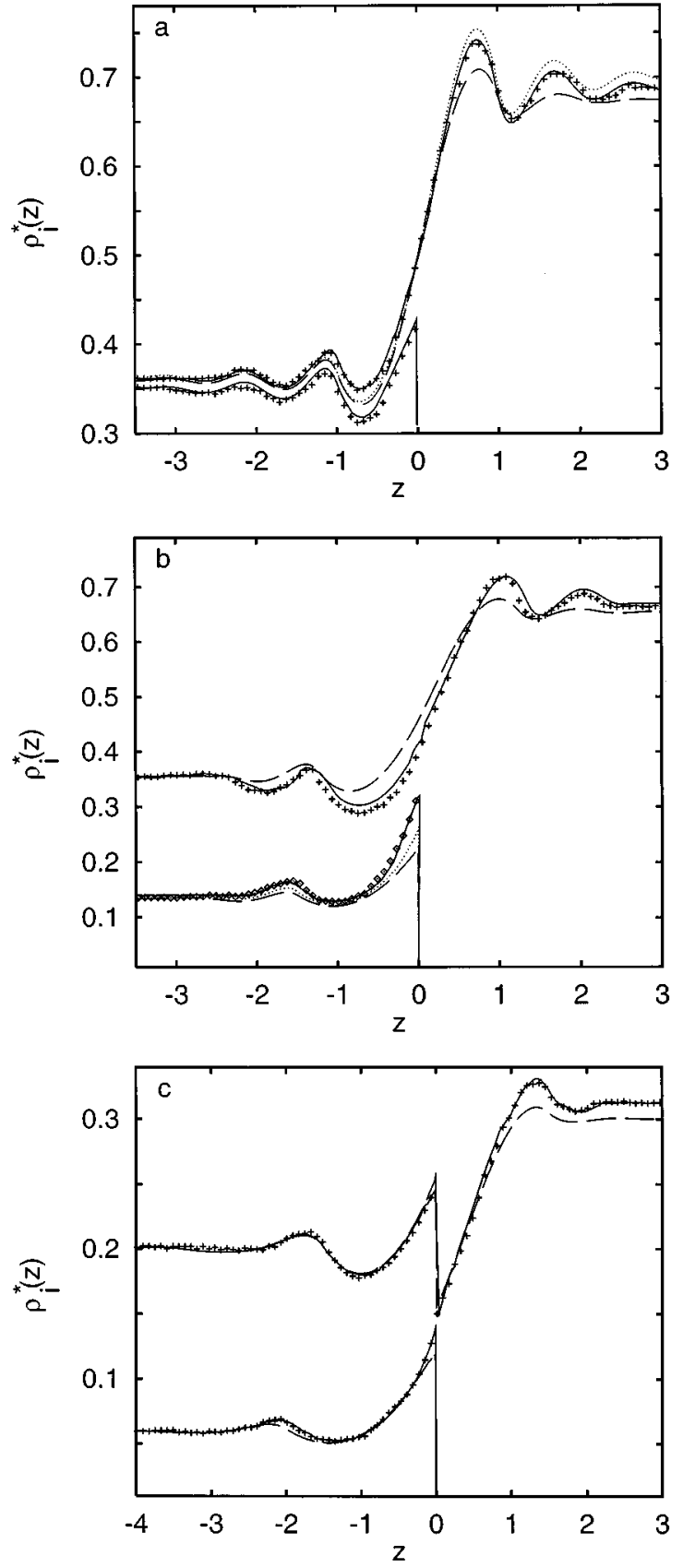

FIG. 1. The density profiles, $\rho_{i}^{*}(z)=\rho_{i}(z) \sigma_{b b}^{3}$, of the nonpermeable, $i$ $=a$, and the permeable, $i=b$, components. Part a is for $\sigma_{a a} / \sigma_{b b}=1$, $\rho_{a} \sigma_{a a}^{3}=0.3532, \quad \rho_{b} \sigma_{b b}^{3}=0.3601$, part $\mathrm{b}$ is for $\sigma_{a a} / \sigma_{b b}=1.5, \rho_{a} \sigma_{a a}^{3}$ $=0.4542\left(\rho_{a} \sigma_{b b}^{3}=0.1346\right), \rho_{b} \sigma_{b b}^{3}=0.3648$, and part $\mathrm{c}$ is for $\sigma_{a a} / \sigma_{b b}=2$, $\rho_{a} \sigma_{a a}^{3}=0.4767,\left(\rho_{a} \sigma_{b b}^{3}=0.05959\right), \rho_{b} \sigma_{b b}^{3}=0.2051$. The local density plots in part $c$ for $z>0$ have been shifted down by 0.2 . The nomenclature of the lines is the following: the solid lines denote the results of the PY2 theory, the dashed line denotes the PY1 approximation, and the dotted line denotes the results of the $\mathrm{HNC1}$ approximation. The points are the Monte Carlo results. To make the figures more clear, only some of the $\mathrm{HNC1}$ results have been displayed.

Further, it is of interest to study more sophisticated interparticle forces and, more generally, the case of associating (or chemically reacting fluids). Such investigations are now in progress in our laboratory.

An alternative approach to the study of nonuniform flu-
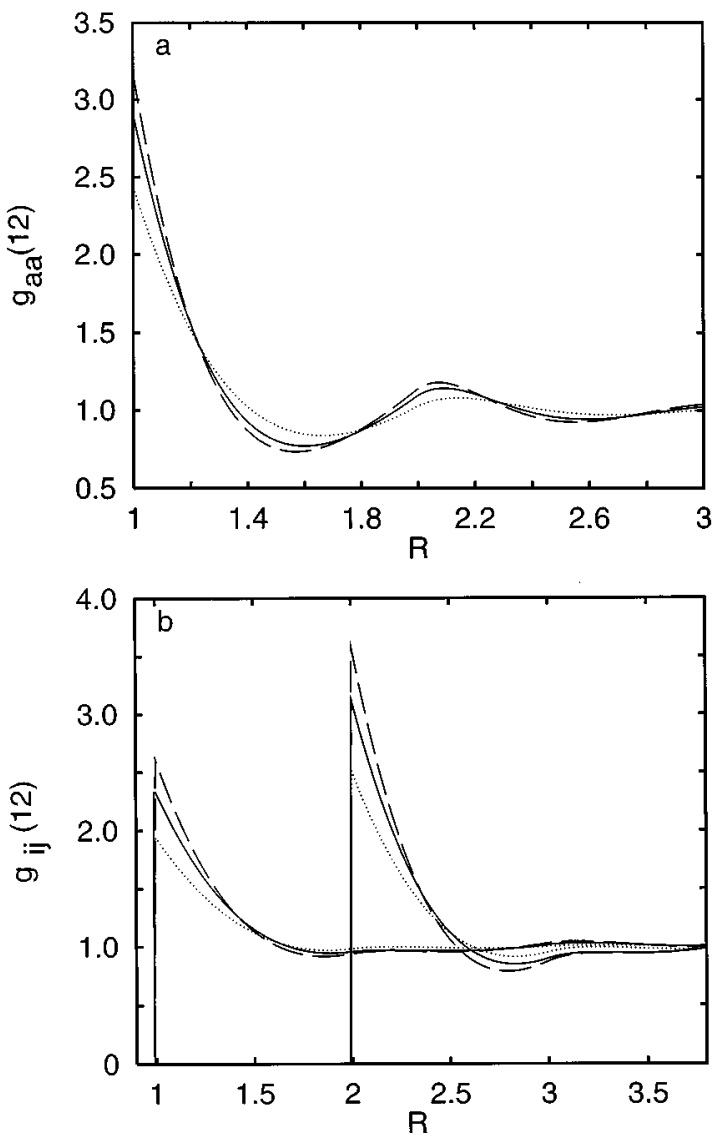

FIG. 2. The inhomogeneous pair distribution functions of the nonpermeable component. The solid line denotes the bulk pair correlation function; the dashed line is the function $g\left(z_{1}=0, z_{2}, R_{12}=0\right)$, i.e., for a perpendicular configuration of both particles, whereas the dashed line denotes the function $g\left(z_{1}=0, z_{2}=0, R_{12}\right)$, i.e., the function characterizing the correlations between two particles in the membrane plane. Part a is for the system shown in Fig. 1(a), whereas part b is for the system displayed in Fig. 1(c).

ids is the density functional approach. It is expected that this approach will be less computer intensive than that used here. The version of Rosenfeld-Kierlik-Rosinberg ${ }^{23,24}$ seems especially promising. This method is under investigation in our laboratory.

The financial support of the U.S. National Science Foundation (Grant No. CHE96-01971) and the Petroleum Research Fund of the American Chemical Society (Grant No. ACS-PRF-31573-AC9) is acknowledged with thanks. Also P.B. wishes to express gratitude to the KBN of Poland for its financial support of this project. D.H. is a John Simon Guggenheim Memorial Foundation Fellow.

${ }^{1}$ D. Henderson, in Fundamentals of Inhomogeneous Fluids, edited by D. Henderson (Marcel Dekker, New York, 1992), Chap. 4.

${ }^{2}$ S. Sokolowski, J. Chem. Phys. 73, 3507 (1980).

${ }^{3}$ S. Sokolowski, Mol. Phys. 49, 1481 (1983).

${ }^{4}$ D. Henderson and M. Plischke, Proc. R. Soc. London, Ser. A 400, 163 (1985).

${ }^{5}$ P. Attard, J. Chem. Phys. 91, 3072 (1989).

${ }^{6} \mathrm{R}$. Evans, in Fundamentals of Inhomogeneous Fluids, edited by D. Henderson (Marcel Dekker, New York, 1992), Chap. 3.

${ }^{7}$ M. Plischke and D. Henderson, J. Chem. Phys. 84, 2846 (1986).

${ }^{8}$ D. Henderson, O. Pizio, and S. Sokolowski, Mol. Phys. (submitted). 
${ }^{9}$ D. Henderson, S. Sokolowski, and A. Trokchymchuk, Phys. Rev. E 52, 3260 (1995).

${ }^{10}$ E. Bruno, C. Caccamo, and P. Tarazona, Phys. Rev. A 35, 1210 (1987).

${ }^{11}$ P. Marsh, G. Rickaysen, and M. Calleja, Mol. Phys. 84, 799 (1995).

${ }^{12}$ J. G. Powles and M. Pagoda, Mol. Phys. 78, 757 (1993).

${ }^{13}$ J. G. Powles, S. Murad, and P. Ravi, Chem. Phys. Lett. 188, 21 (1992).

${ }^{14}$ D. Henderson, A. Trokhymchuk, and O. Pizio, Chem. Phys. Lett. 245, 615 (1995).

${ }^{15}$ Y. Zhou and G. Stell, J. Chem. Phys. 89, 7010, (1988).

${ }^{16}$ Y. Zhou and G. Stell, J. Chem. Phys. 89, 7020, (1988).
${ }^{17}$ W. J. Ellis, Mol. Phys. 82, 973 (1994).

${ }^{18}$ T. L. Hill, An Introduction to Statistical Thermodynamics (AddisonWesley, Reading, 1960).

${ }^{19}$ K. S. Cole, Membranes, Ions and Impulses (University of California Press, Berkeley, 1968).

${ }^{20}$ R. Lovett, I. Mou, and F. P. Buff, J. Chem. Phys. 65, 570 (1976).

${ }^{21}$ S. Labík, A. Malijevský, and P. Vonka, Mol. Phys. 56, 709 (1985).

${ }^{22}$ P. Bryk, O. Pizio, S. Sokolowski, Mol. Phys. (in press).

${ }^{23}$ Y. Rosenfeld, Phys. Rev. Lett. 63, 980 (1989).

${ }^{24}$ E. Kielik and M. L. Rosinberg, Phys. Rev. A 42, 3382 (1990). 